\title{
Solar Optical Telescope onboard Hinode for Diagnosing the Solar Magnetic Fields
}

\author{
Kiyoshi ICHIMOTO and Hinode/SOT team \\ National Astronomical Observatory/NINS, Mitaka, Tokyo 181-8588, Japan
}

(Received 13 December 2006 / Accepted 15 May 2007)

\begin{abstract}
The Solar Optical Telescope (SOT) onboard Hinode is a $50 \mathrm{~cm}$ aperture telescope to observe the sun in visible lights $(388-668 \mathrm{~nm})$ for the first time with high and extremely stable spatial resolution from space. The focal plane package of SOT consists of Broad-band Filter Imager which provides the highest spatial resolution images of the solar photosphere, the Narrow-band Filter Imager which takes 2-dimentional Dopplergram and Magnetogram of photosphere and chromosphere in high cadence and with wide field of view, and the Spectro-Polarimeter which takes full Stokes line profiles to provide the highest precision magnetic field maps of the photosphere. The most outstanding characteristics of SOT is its continuous and uniform data quality with the unprecedentedly high precision and high spatial resolution of 0.2-0.3 arcsec.

The door of the telescope was deployed on 25th Oct. followed by a successful SOT first light. Initial checkouts of the instrument were conducted and superior performance of SOT was confirmed. SOT is starting the regular observation of the daily target region on the sun and producing excellent scientific data. Overview of the SOT instrument is summarized and initial results are demonstrated.
\end{abstract}

(c) 2007 The Japan Society of Plasma Science and Nuclear Fusion Research

Keywords: optical telescope, sun, solar atmosphere, magnetic field, polarization, spectroscopy

DOI: $10.1585 / \mathrm{pfr} .2 . S 1009$

\section{Introduction}

The solar atmosphere is highly magnetized plasma revealing various type of active phenomena like mass ejection (jets), sudden eruption of prominence (cool material suspended in the corona), vital explosion called as flare, and so on. The very existence of the hot and tenuous atmosphere, corona, is one of the long-standing mystery in astrophysics. It is recognized from the recent groundbased observations that the magnetic fields which drives the active phenomena in the corona is highly structured at the photospheric level with a spatial scale of $0.2 \operatorname{arcsec}$ or less [1], and high resolution observations of fundamental process in solar photosphere is of a crucial importance to understand the dynamics of the solar plasma. This was the driving force for us to start the Solar-B project in early 1990th.

The Solar Optical Telescope (SOT) onboard Hinode is aimed to study fundamental processes taking place on the solar surface and to provide definite boundary conditions that drive the various active phenomena observed in the outer solar atmosphere (corona) [2,3]. Following the successful launch of Hinode satellite on 23rd Sep. 2006, the top door of the telescope was deployed on 25th Oct. 2006 and we met very successful first light immediately. Thanks to the sun-synchronous orbit of Hinode, SOT is now providing continuous observation of the selected target region on the sun, and sending us unprecedentedly high quality images.

In this paper, we describe the overview of the SOT instrument, its performance in orbit, and demonstrate some of initial results.

\section{The SOT Instrument}

\subsection{Overview of the optical system}

The SOT consists of the Optical Telescope Assembly (OTA) [4] which is an aplanatic Gregorian telescope with an aperture diameter of $500 \mathrm{~mm}$, and the Focal Plane Package (FPP) which produces scientific data with the filtergraph and spectrograph instruments. Fig. 1 shows the optical layout of SOT. The OTA provides a unaberrated solar image to the FPP with a diffraction limited wavefront quality (Strehl $>0.8$ at $500 \mathrm{~nm}$ ). The distance between primary and secondary mirrors is $1500 \mathrm{~mm}$ and the linear central obscuration is 0.344. The Heat Dump Mirror (HDM) located at the primary focus of OTA is a $45 \mathrm{deg}$. flat mirror with a central hole, and it reflects about $88 \%$ of incident solar energy (the lights outside of the SOT field of view) out to the space through a side window of the OTA. The Collimating Lens Unit (CLU) after the secondary focus of the Gregorian telescope provides collimated beam with a minimized chromatic aberration. The Polarization Modulator Unit (PMU) located after the CLU is a rotating linear waveplate to provide efficient polarization modulations at wavelengths for vector magnetic field measurement.

With the successive tip-tilt folding mirror, OTA send 


\section{Optical layout of SOT}

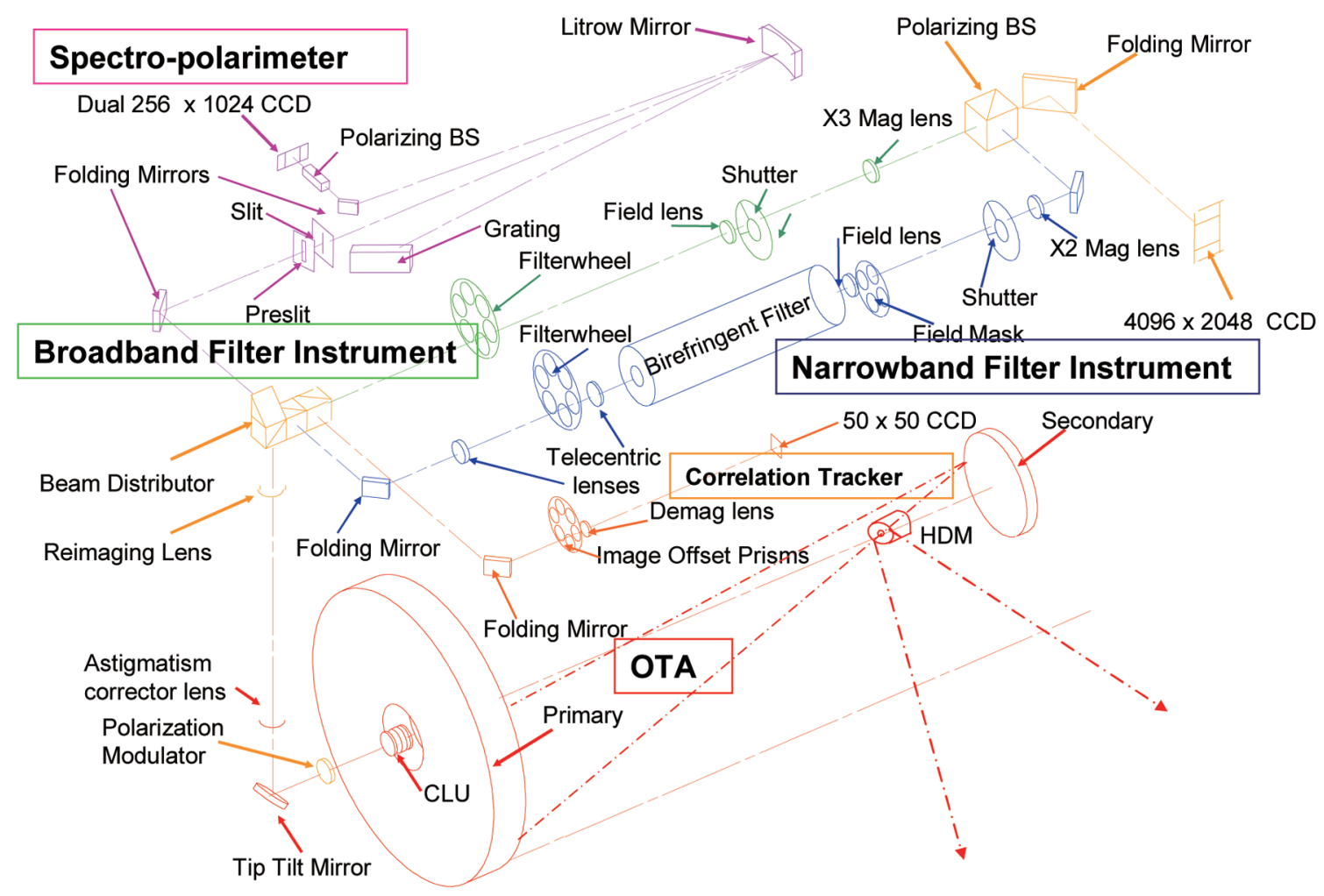

Fig. 1 Optical layout of SOT.

Table 1 Basic parameters of four optical path.

\begin{tabular}{|l|c|c|c|c|}
\hline & BFI & NFI & SP & CT \\
\hline CCD format & \multicolumn{2}{|c|}{$4096 \times 2048$} & $112 \times 1024 \times 2$ & $50 \times 50$ \\
\hline pixel scale (arcsec/pix) & 0.054 & 0.08 & 0.16 & 0.22 \\
\hline $\begin{array}{c}\text { maximum FOV (arcsec }{ }^{2} \text { ) } \\
\text { (EWxNS) }\end{array}$ & $218 \times 109$ & $328 \times 164$ & $\begin{array}{c}328 \text { (scan range) } \\
\text { x164 (slit length) }\end{array}$ & $11 \times 11$ \\
\hline wavelength resolution (A) & $3 \sim 10$ & $\sim 0.1$ & 0.02 & 5 \\
\hline $\begin{array}{l}\text { number of wavelength in a } \\
\text { data set }\end{array}$ & 1 & $1 \sim 4$ & 244 & 1 \\
\hline time resolution (typical) & $5 \sim 30 \mathrm{~s}$ & $10 \sim 60 \mathrm{~s}$ & $1 \mathrm{~min} \sim 3 \mathrm{hr}$ & $580 \mathrm{~Hz}$ \\
\hline photometric aquracy (\%) & 0.5 & $0.1 \sim 0.5$ & $\sim 0.1$ & $\sim 0.5$ \\
\hline
\end{tabular}

the pointing stabilized beam to the FPP. The diameter of HDM limits the allowable maximum offset pointing angle of SOT (or Solar-B) from the sun center; which is 19.6 arcmin (for details of OTA, see Ichimoto etal. 2005 and Shimizu 2004).

FPP has four observing paths; Narrowband Filter Imager (NFI), Broadband Filter Imager (BFI), Spectropolarimeter (SP) and Correlation Tracker (CT). The entrance of FPP is the reimaging lens which refocus the solar image inside the FPP. A focus error of SOT after the launch is eliminated by adjusting the position of the reimaging lens in orbit. The beam splitter assembly following the reimaging lens distributes the beam from OTA to four optical paths in FPP. Basic instrumental parameters of the four observing paths are summarized in Table 1, while the observ-
Table 2 Observing wavelengths of SOT.

\begin{tabular}{|l|c|l|c|c|c|c|c|}
\hline Ion & $\boldsymbol{\lambda}, \mathbf{n m}$ & \multicolumn{1}{c|}{ Purpose } & $\mathrm{g}_{\text {eff }}$ & BFI & NFI & SP & CT \\
\hline CN I & 388.30 & Magnetic Network Imaging & - & $\checkmark$ & & & \\
\hline Ca II H & 396.85 & Chromospheric Heating & 1.33 & $\checkmark$ & & & \\
\hline CH I & 430.50 & Magnetic Elements & - & $\checkmark$ & & & \\
\hline & 450.45 & Blue Continuum & & $\checkmark$ & & & \\
\hline Mg I b & 517.27 & Chromospheric Dopp./Mag. & 1.75 & & $\checkmark$ & & \\
\hline Fe I & 524.71 & Photospheric Magnetograms & 2.00 & & $\checkmark$ & & \\
\hline Fe I & 525.02 & Photospheric Magnetograms & 3.00 & & $\checkmark$ & & \\
\hline Fe I & 525.06 & Photospheric Magnetograms & 1.50 & & $\checkmark$ & & \\
\hline & 555.05 & Green Continuum & & $\checkmark$ & & & \\
\hline Fe I & 557.61 & Photospheric Dopplergrams & 0.00 & & $\checkmark$ & & \\
\hline Na D & 589.59 & Chromospheric Dopp./Mag. & & & $\checkmark$ & & \\
\hline Fe I & 630.15 & Photospheric Magnetograms & 1.67 & & $\checkmark$ & $\checkmark$ & \\
\hline Fe I & 630.25 & Photospheric Magnetograms & 2.50 & & $\checkmark$ & $\checkmark$ & \\
\hline Ti I & 630.38 & Umbral Magnetograms & 0.92 & & $\checkmark$ & & \\
\hline & 632.00 & Broadband WL for CT & - & & & & $\checkmark$ \\
\hline H I & 656.28 & Chromospheric Structure & - & & $\checkmark$ & & \\
\hline & 668.40 & Red Continuum & & $\checkmark$ & & & \\
\hline
\end{tabular}

ing wavelengths and their scientific aim are summarized in Table 2.

NFI takes vector magnetograms and Dopplergrams in selected spectral lines and $\mathrm{H} \alpha$ images by using a tunable Lyot-type filter. The tunable filter has a bandpass of approximately $0.1 \mathrm{~A}$, and is located in a $\mathrm{F} / 31$ telecentric beam. BFI takes photospheric images in the highest spatial resolution with a spatial sampling of $0.054 \mathrm{arcsec} / \mathrm{pixel}$. A 
common CCD with a format of $4096 \times 2048$ pixels is used for NFI and BFI. SP takes high precision full Stokes profiles in magnetically sensitive FeI lines at $630.25 \mathrm{~A}$ and $630.15 \mathrm{~nm}$. By tilting a folding mirror in the SP path, the solar image on the slit is shifted in east-west direction with a step of 0.16 arcsec and a range of 328 arcsec to take two dimensional map. CT produces a pointing error signal at $580 \mathrm{~Hz}$ by taking the cross-correlation of granulation images, and the signal is used to control the tip-tilt mirror in OTA to stabilize the solar image on CCDs. By a closed loop control of $\mathrm{CT}$ and tip-tilt mirror, the pointing stability of $<0.03$ arcsec rms is achieved [5].

\subsection{Data products}

The PMU is a continuously rotating linear waveplate with a rate of $1.6 \mathrm{sec} / \mathrm{rev}$. Its retardance is designed to produce an efficient polarization modulation in boh linear and circular polarization at the wavelengths for vector magnetic field measurement at FeI $630 \mathrm{~nm}$ and $\mathrm{MgI} 517 \mathrm{~nm}$.

SP takes 16 images in every rotation of PMU with a fixed exposure length of $0.1 \mathrm{sec}$. By the processing of the successive frames (addition and subtraction), a full set of Stokes parameters of the pair of the FeI spectral lines at $630.15 \mathrm{~nm}$ and $630.25 \mathrm{~nm}$ is accumulated on board. SP takes both of orthogonally polarized spectra simultaneously to mitigate polarization noise induced by residual image motions during the sampling. Typical onboard integration time is for PMU 3 rotations $(4.8 \mathrm{sec})$ for each slit position and a photometric accuracy is about 0.001 in continuum.

For NFI, a mechanical shutter can be used to isolate the exposure duration against the phase of PMU. In this shutter-mode, full field of view observation is possible with a certain cost of the modulation speed and photometric accuracy. The maximum exposure length is $0.4 \mathrm{sec}$ for $\mathrm{V}$ measurement and $0.2 \mathrm{sec}$ for $\mathrm{Q}$ and $\mathrm{U}$ measurements. The scheme of exposure sequence to obtain a set of polarized images is flexibly controlled by the software. NFI has a focal plane mask wheel with which the field of view is restricted to enable a continuous readout of $\mathrm{CCD}$ in synchronous with the PMU without using the mechanical shutter. In this shutterless-mode, the NFI takes $16 \mathrm{im}-$ ages in every revolution of PMU as SP, and onboard accumulation and demodulation are performed to achieve the photometric accuracy of $\sim 0.001$. The selectable field of view with the mask wheel is $16^{\prime \prime}, 32^{\prime \prime}, 64^{\prime \prime} \times 163.8^{\prime \prime}$ corresponding $1 \times 1,2 \times 2$ and $4 \times 4$ pixel binning.

The BFI provides simple wide field of view images with the mechanical shutter. Timing of exposures with respect to the PMU angle are again selectable.

The sequence of SOT observation is conducted by so called 'observation tables' stored in the Mission Data Processor (MDP) onboard Hinode satellite. We can make a quite flexible planning of observation regarding the combination of observables, time cadence, field of view, etc., within the constraint of the allocated data rate. The data is compressed by MDP using the algorithm of DPCM or JPEG, where the quality (compression rate) is selectable for JPEG. Thanks to the Svalbard Station which carries 15 downlinks of the mission data every day, SOT can take the data of about $10 \mathrm{~GB} /$ day after decompression at this point. The planning is conducted in daily basis in collaboration with XRT and EIS instrument teams.

\section{Instrument Checkout}

After the deployment of the side door of OTA on 14th October 2006, electrical components of SOT were turned on and basic functional tests of moving mechanisms and cameras were carried out successfully. The period after opening the side door and before opening the top door is designated as the initial bakeout phase of the OTA prior to introducing the sun light into the telescope, and decontamination heaters of the primary and secondary mirrors of OTA were turned on to maintain the temperature of critical surfaces higher than other parts of the telescope. A number of dark images of SP and FG were obtained in this period for forthcoming data reduction.

The top door of OTA was deployed on 25th October with the successful first light followed immediately. The position of the reimaging lens for the best focus was determined and we confirmed that it is fairly close to the prediction from the pre-launch ground testing. The first light image in BFI/G-band (Figure 2) after the initial focus adjustment already reveals the diffraction limited performance of SOT.

Image stabilization system consisting of CT and tiptilt mirror is in operation after Hinode started the tracking of target regions on the sun by compensating the solar rotation. Figure 3 shows the time profiles of pointing error produced by CT. An excellent performance of the image stabilization system is obvious with the achieved image stability of $\sim 0.01$ arcsec rms (three times better than the requirement). More quantitative results of the image quality and CT performance will be described in separate papers.

Basic performance of the instruments, i.e., image
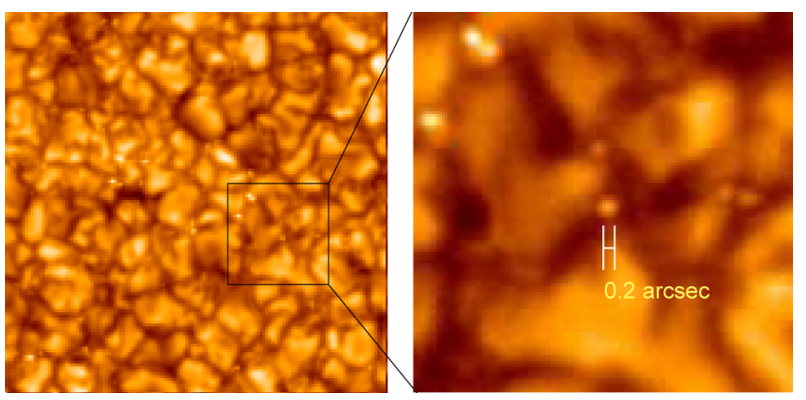

Fig. 2 G-band $(430.5 \mathrm{~nm})$ image of quiet region with BFI. The size of the smallest feature is about $0.2 \operatorname{arcsec}(\sim 140 \mathrm{~km})$ which correspods to the diffraction limit of this wavelength with $50 \mathrm{~cm}$ aperture. 


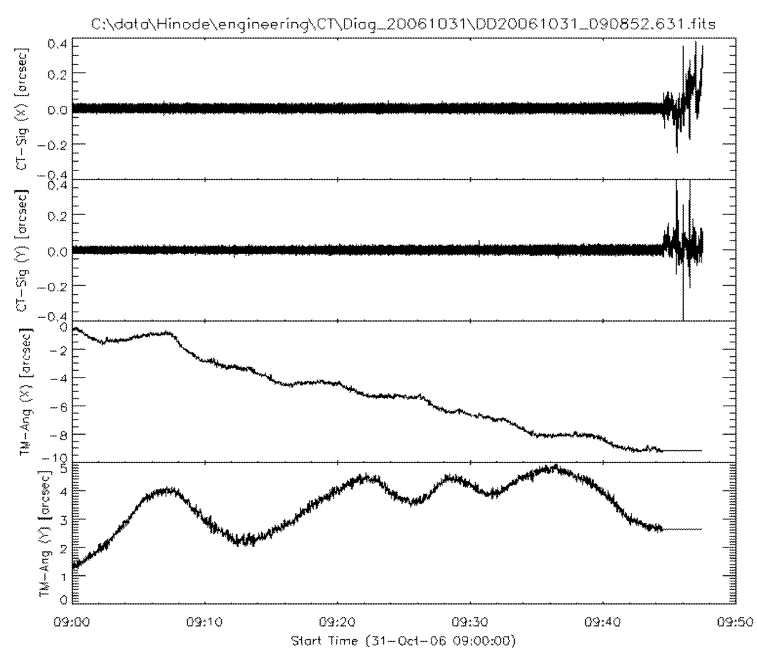

Fig. 3 Pointing error (signal from CT at $580 \mathrm{~Hz}$; top and second plots) and angle of tip-tilt mirror (third and bottom plots). Around 9:44, CT servo control was turned off. The pointing error is about 0.01 arcsec rms during CT is in operation.

quality, vignetting, light level and so on, were confirmed to be fine as expected for all wavelengths of BFI and SP. It is also confirmed that the NFI provides excellent longitudinal magnetogram in FeI $630.25 \mathrm{~nm}$ line. However there found unexpected degradation of a part of the NFI field of view due to a shade created in the tunable filter after launch. By this reason, the NFI is operated at this point under the limited function, and engineering checkout is still on-going to find the way of operating the tunable filter with minimum influence of the artifacts in NFI observation.

\section{First Light Images}

Figure 4 shows the G-band and CaII H images of quiet sun near the disk center taken by BFI. Granulation patterns (convection cells in the photosphere) are obvious in G-band image and their contrast is reversed in CaII H image, which may provide valuable insight for the study of chromospheric heating. There noticed a number of pointlike bright features in the intergranular lane in both G-band and $\mathrm{CaII} \mathrm{H}$ images. These structures are considered as the elementary magnetic field on the sun, and precise study of their behavior are thought to be of crucial importance for understanding the heating and dynamics of the upper atmosphere. The movie with uniform image quality provided for the first time by SOT reveals that each bright point is wobbled by granules convective motion and injections of kinetic energy into the magnetic field is certainly taking place which may be transported to the upper atmosphere.

Figure 5 shows sunspot images in G-band and CaII $\mathrm{H}$ by $\mathrm{BFI}$ and a magnetogram (circular polarization map in blue wing of FeI $630.25 \mathrm{~nm}$ ) taken by NFI. The filamentary structure in sunspot penumbra is clearly visible in both G-band and CaII $\mathrm{H}$ images and also in the magnetogram.

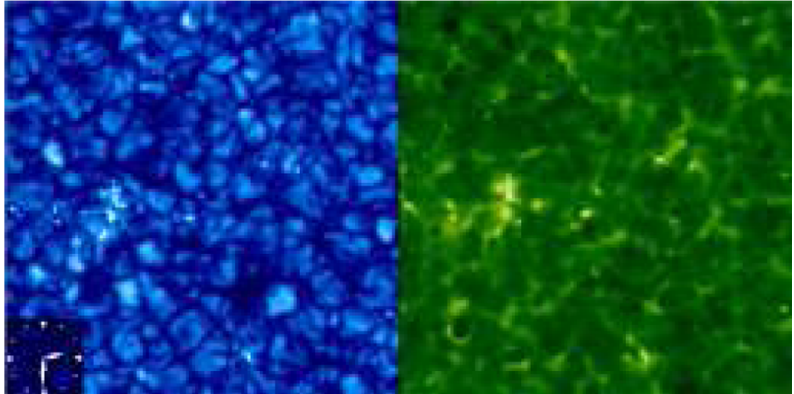

Fig. 4 G-band $(430.5 \mathrm{~nm})$ and CaII H $(396.8 \mathrm{~nm})$ images of quiet sun near the disk center taken by BFI.
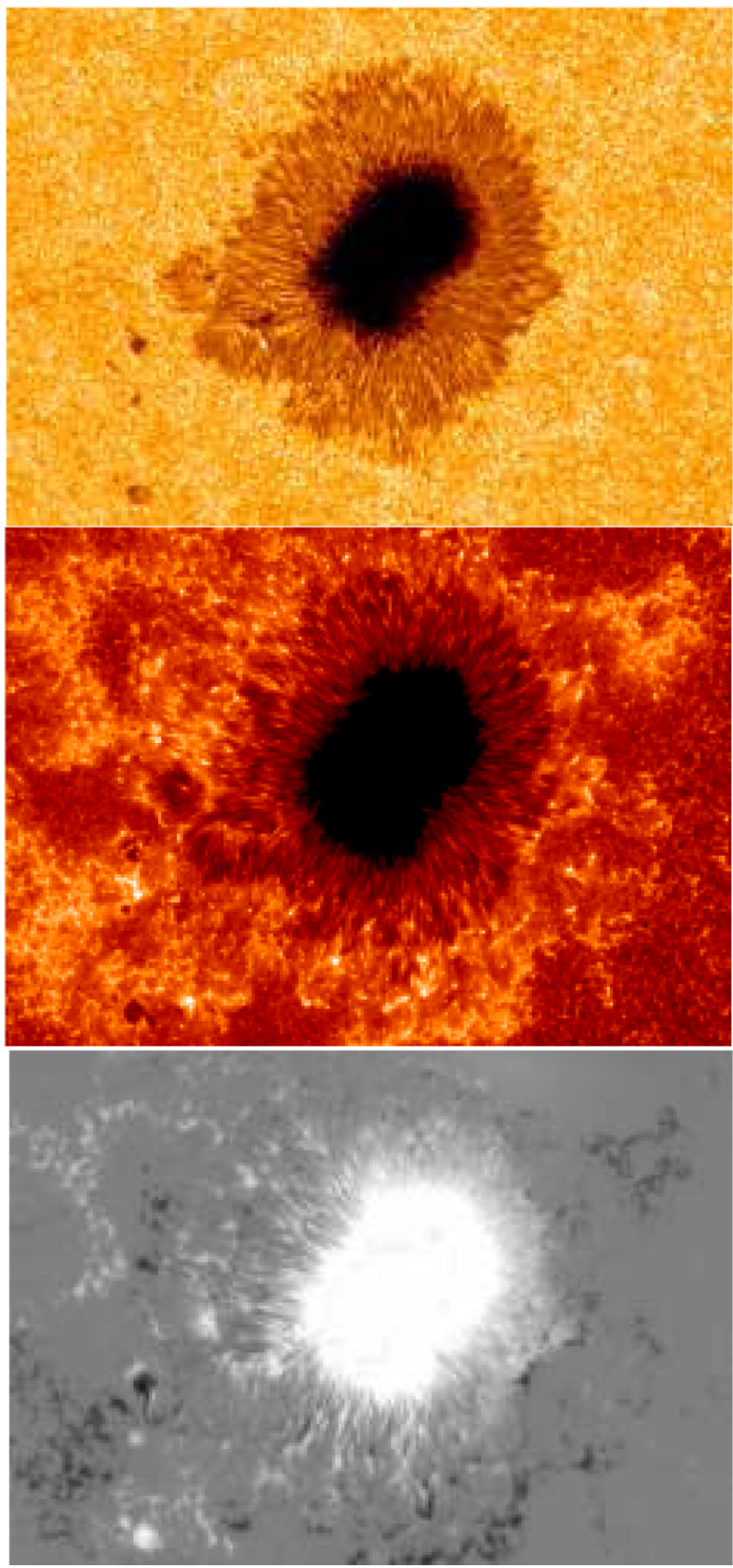

Fig. 5 Sunspot image in G-band $(430.5 \mathrm{~nm})$ and CaII H $(396.8 \mathrm{~nm})$ by BFI and magnetogram by NFI. 


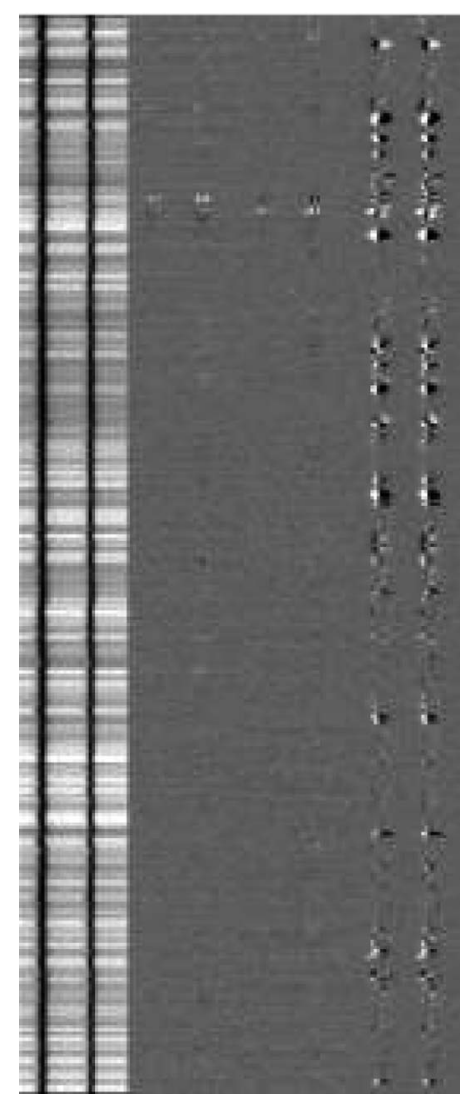

Fig. 6 Example of Stokes spectra taken by SP on the quiet region near the disk center. From left to right shown the Stokes I, Q, U and V spectra; horizontal axis is wavelength covering two FeI lines at 630.15 and $630.25 \mathrm{~nm}$, while vertical axis is distance along the slit.

It should be emphasized that the uniformity of the SOT image quality is a great advantage for obtaining an accurate magnetogram which is composed from multiple images. The correlation between the intensity and magnetic fields can be studied precisely for the fine scale structure of penumbra for the first time.

Figure 6 shows an example of SP data which is taken with the slit located on a quiet region near the disk center. From left to right shown the Stokes I, Q, U and V spectra; horizontal axis is wavelength covering two FeI lines at 630.15 and $630.25 \mathrm{~nm}$, while vertical axis is distance along the slit. A number of localized polarization signals are evident even in the quiet sun and we noticed some of them show striking red shift especially in Stokes V which implies dynamical nature of the flux tubes. Figure 7 shows 2D maps of quiet region in continuum intensity and Stokes $\mathrm{V}$ in blue wing of FeI $630.25 \mathrm{~A}$ line made from a set of scan data by SP. These example reveals the excellent performance of SP for diagnosing the solar magnetic fields.

Figure 8 shows a CaII $\mathrm{H}$ image at the limb. There exists a sunspot just on the limb and we can see a number of structures extending from the limb. They are likely the traces of magnetic fields in the corona. In the movie

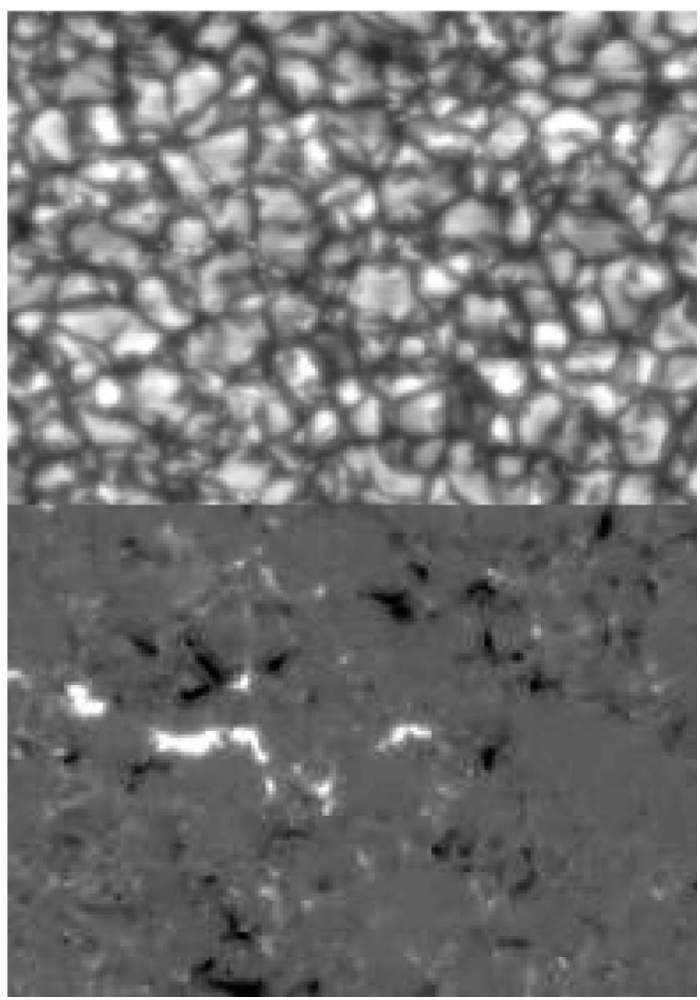

Fig. 7 Map of quiet region in continuum intensity and Stokes V in blue wing of FeI $630.25 \mathrm{~nm}$ line made from a set of scan data by SP.

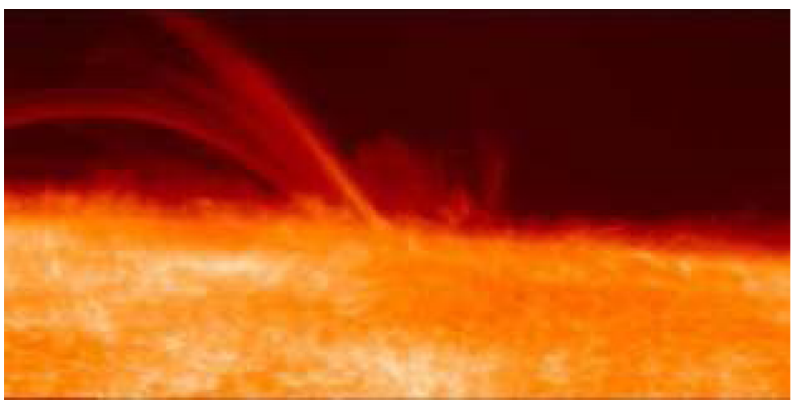

Fig. 8 CaII H (396.8 nm) image at the solar limb.

(http://hinode.nao.ac.jp/index_e.shtm), we can recognize a spectacular scenery showing expanding, flowing and falling motions of structures, and they certainly provide new insight on the dynamics of the solar corona. Such new scenery became available for the first time again owing to the uniform image quality of SOT.

\section{Summary}

The SOT is the largest telescope ever built to observe the Sun from space. It provides diffraction limited images (0.2-0.3 arcsec) continuously under the condition free from the atmospheric seeing. Thanks to the sun-synchronous orbit of Hinode, SOT can provides continuous observation for $24 \mathrm{hr}$ per day in $\sim 8$ months per year. Hinode/SOT is now providing excellent images of solar photosphere and 
chromosphere ( $\sim 10 \mathrm{~GB} /$ day $)$; the BFI and SP are in operation with their full performance, NFI is in operation but with limited wavelength tuning; engineering checkout is still ongoing.

It is promising that Hinode/SOT will provide over the next 3-10 years unprecidented observations of the magnetic field evolution in the photosphere, and, together with the XRT/EIS observations, Hinode will open the new world in the plasma physics of the solar atmosphere.

\section{Acknowledgement}

The SOT team members are S. Tsuneta, Y. Suematsu, Y. Katsukawa, M. Noguchi, M. Nakagiri, S. Kamio (NAOJ), T. Shimizu, M. Kubo (JAXA/ISAS), T. Tarbell, R. Shine, A. Title, T. Berger (LMSAL), B. Lites, D. El- more (HAO), T. Okamoto, S. Nagata (Kyoto-Univ.). SOT is a joint program between JAXA and NASA. The author likes to express his deep thanks to late Professor T. Kosugi of ISAS and Dr. L. Hill and J. Owens of NASA for continuous support of the development of SOT. OTA was constructed by Mitsubishi Electrical Corporation and FPP was built by Lockheed Martin Corporation.

[1] J. Stenflo, Solar Magnetic Field (2001).

[2] Y. Suematsu and the Solar-B Team., ASP conference series 289, 37 (2003).

[3] T. Shimizu., ASP conference series 325, 3 (2004).

[4] K. Ichimoto et al., SPIE conference series 5487, 1142 (2004).

[5] T. Shimizu et al., SPIE conference series 5487, 1152 (2004). 